\title{
Detection of holes in an elastic body based on eigenvalues and traces of eigenmodes
}

\author{
Pedro R.S. Antunes ${ }^{a}$, Cristian Barbarosie ${ }^{b}$, Anca-Maria Toader $^{b}$ \\ a Grupo de Física Matemática da Universidade de Lisboa, Faculdade de Ciências da Universidade de Lisboa, Campo Grande, Edifício C6, \\ 1749-017 Lisboa, Portugal \\ ${ }^{\mathrm{b}}$ CMAF-CIO, Faculdade de Ciências da Universidade de Lisboa, Campo Grande, Edifício C6, Piso 1, 1749-016 Lisboa, Portugal
}

\section{A R T I C L E I N F O}

\section{Article history:}

Received 28 July 2016

Accepted 21 December 2016

Available online 28 December 2016

\section{Keywords:}

Inverse problem

Shape optimization

Eigenvalue problem

Linear elasticity

Adjoint method

Method of fundamental solutions

\begin{abstract}
A B S T R A C T
We consider the numerical solution of an inverse problem of finding the shape and location of holes in an elastic body. The problem is solved by minimizing a functional depending on the eigenvalues and traces of corresponding eigenmodes. We use the adjoint method to calculate the shape derivative of this functional. The optimization is performed by BFGS, using a genetic algorithm as a preprocessor and the Method of Fundamental Solutions as a solver for the direct problem. We address several numerical simulations that illustrate the good performance of the method.
\end{abstract}

(C) 2016 Elsevier Inc. All rights reserved.

\section{Introduction}

The goal of this paper is to develop an inverse method, both analytical and numerical, for detecting the shape of unknown holes inside an elastic body. Free vibrations of this body are considered. Physical measurements on the eigenvalues and on the traces of the eigenmodes on a certain part of the exterior boundary are used as input data in the process of recovering the shape(s) of the hole(s).

The existing approaches in the literature focus on matching the measured eigenvalues (see e.g. $[14,15,20]$ ) but the information coming from the eigenmodes is not taken into account. An exception is [21] where measurements on eigenmodes are used in order to detect damaged zones in a concrete dam, by a free material approach.

In the present work the shapes of the holes are recovered through a shape optimization procedure, namely, by minimizing the objective functional (3) in Section 2, which is a least square distance between the computed eigenvalues/eigenmodes and the measured ones.

In Section 3, the shape derivative of the objective functional is computed, which requires the shape derivatives of the eigenvalues and eigenmodes. An essential tool is the adjoint method which has been adapted to the current framework. To our knowledge, this is the first time when the shape derivative of a functional depending on eigenmodes is computed.

The direct problem, that is, the computation of eigenvalues and eigenmodes for given shape(s) of hole(s), is done through the Method of Fundamental Solutions (MFS), see Section 4. This is a meshless method which builds the solution as a convolution of fundamental solutions centered at chosen points located outside the domain. This ensures that the state equation

\footnotetext{
ty P.A. is partially supported by FCT, Portugal, through the Program "Investigador FCT" with reference IF/00177/2013 and the scientific project PTDC/MATCAL/4334/2014. C.B. and A.-M.T. are supported by Fundação para a Ciência e a Tecnologia, UID/MAT/04561/2013.

E-mail addresses: prantunes@fc.ul.pt (P.R.S. Antunes), cabarbarosie@fc.ul.pt (C. Barbarosie), atoader@fc.ul.pt (A.-M. Toader).
} 


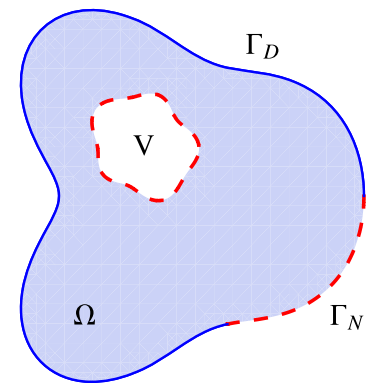

Fig. 1. The boundaries for the problem of detecting an inclusion $V$.

is satisfied exactly, while the boundary conditions will be satisfied only approximately. Choosing an initial guess turns out to be a delicate question since our approach does not allow for topology changes during the optimization procedure. To avoid these difficulties, at a first step we use a genetic algorithm to provide a good initial guess.

In Section 5, three numerical examples are presented. The first one consists in detecting the shape of one hole included in a square elastic body with measurements of five eigenvalues and eigenmodes on one side of the square. In the second example three holes are to be detected in a rectangular body having measurements of five eigenvalues and the traces of the eigenmodes on three sides of the rectangle. In the last example we consider the same domain as in example 2, but use just one eigenvalue and the measurements of the trace of the corresponding eigenmode on a smaller part of the boundary.

\section{Problem formulation}

Let $\Omega \subset \mathbb{R}^{2}$ be a bounded domain. The boundary is made of two disjoint parts $\Gamma_{D}$ and $\Gamma_{N}$, respectively with Dirichlet and Neumann boundary conditions. Denote by $V \subset \Omega$ an inclusion which is unknown (Fig. 1).

Given the specific mass $\rho$ and the elastic tensor $C$, we consider the eigenvalue problem

$$
\left\{\begin{array}{l}
\nabla \cdot \sigma(u)+\rho \Lambda u=0 \quad \text { in } \Omega \backslash V, \\
u=0, \quad \text { on } \Gamma_{D}, \\
\sigma(u) n=0 \text { on } \Gamma_{N} \cup \partial V,
\end{array}\right.
$$

where

$$
\sigma(u)=C \epsilon(u)
$$

and

$$
\epsilon(u)=\frac{1}{2}\left(\nabla u+\nabla u^{T}\right) .
$$

On the current domain $\Omega \backslash V$, the variational formulation of (1) writes

$$
\int_{\Omega \backslash V} C \epsilon(u) \epsilon(v) d x=\Lambda \int_{\Omega \backslash V} \rho u v d x,
$$

which can be written in component notation as:

$$
\int_{\Omega \backslash V} C_{i j k l} u_{i, j} v_{k, l} d x=\Lambda \int_{\Omega \backslash V} \rho u_{i} v_{i} d x .
$$

In the above, $u_{i, j}$ denotes the partial derivative of the $u_{i}$ component in the direction $x_{j}$. We have used Einstein's summation convention and the symmetry properties of the fourth order tensor $C$. Assuming that the body is isotropic and has the Lamé coefficients $\lambda$ and $\mu$, the elastic tensor is defined by

$$
C \xi=\lambda(\operatorname{tr} \xi) I+2 \mu \xi
$$

for all symmetric matrices $\xi$.

We will denote the eigenvalues by $0<\Lambda^{1}(\Omega) \leq \Lambda^{2}(\Omega) \leq \ldots$ where each $\Lambda^{q}(\Omega)$ is counted with its multiplicity and the corresponding (normalized) eigenfunctions by $u^{q}, q=1,2, \ldots$.

In this context we define the direct problem: Given the inclusion $V$, we want to compute the first $N$ eigensolutions $\left(\Lambda^{q}, u^{q}\right), q=1, \ldots, N$.

On the space $L^{2}(\Omega \backslash V)^{2}$, we shall consider the norm $\|\cdot\|_{L_{\rho}^{2}}$, induced by the following inner product associated to the function $\rho \in L^{\infty}(\Omega \backslash V)$ representing the specific mass $\left(\rho(x) \geq \rho_{0}>0\right.$ almost everywhere in $\left.\Omega \backslash V\right)$ : 


$$
(u, v) \mapsto \int_{\Omega \backslash V} \rho u \cdot v d x
$$

In the inverse problem, we assume that we know the first $N$ eigenvalues $\tilde{\Lambda}^{q}$ and the traces $\tilde{u}^{q}$ of the corresponding eigenfunctions on $\Gamma_{m}, \Gamma_{m}$ being a subset of $\Gamma_{N}$. Given this data we want to recover the shape and size of the hole(s). The quantities $\tilde{\Lambda}^{q}$ and $\tilde{u}^{q}$ are to be measured experimentally; however, in the present work we compute them by solving the direct problem for a certain target inclusion $\tilde{V}$. The optimization process consists in the minimization of the functional

$$
J(\Omega \backslash V)=\sum_{q=1}^{N}\left|\Lambda^{q}-\tilde{\Lambda}^{q}\right|^{2}+\sum_{q=0}^{N}\left\|\left.u^{q}\right|_{\Gamma_{m}}-\left.\tilde{u}^{q}\right|_{\Gamma_{m}}\right\|_{L^{2}\left(\Gamma_{m}\right)}^{2}
$$

Remark 1. Measuring experimentally the quantities $\tilde{\Lambda}^{q}$ and $\tilde{u}^{q}$ is a challenging task in itself. We refer to [21] and references therein for a real world example, a Dynamic Monitoring System developed at Laboratório Nacional de Engenharia Civil (LNEC), installed on Cabril dam (Portugal) in 2008. This monitoring system includes 16 high sensitivity uniaxial accelerometers (located at the upper zone of the dam, for measuring radial accelerations) that measure continuously and accurately vibrations of low amplitude (at a rate of $1000 \mathrm{~Hz}$ ), namely, vibrations due to ambient excitation. The values of natural frequencies and vibration modes are extracted from the measured acceleration records, using Modal Identification techniques on the frequency domain that are mainly based on the Fourier analysis of the radial acceleration records.

Remark 2. The eigenmodes $u^{q}$ are not unique. Even in the case when $\Lambda^{q}$ is simple, $u^{q}$ is an arbitrary representant in the one-dimensional associated eigenspace. It is usual to normalize $u^{q}$ in order to reduce the arbitrariness, but even after doing so we still have two possibilities, $u^{q}$ and $-u^{q}$. See Remark 7 in Section 4 for implementation details.

Remark 3. In the present work, it is straightforward to normalize $\tilde{u}^{q}$ in the $L_{\rho}^{2}(\Omega)$ because it is computed as a solution of a target direct problem. However, in the more realistic case when the trace of $\tilde{u}^{q}$ is obtained through physical measurements, this is impossible since we do not know $\tilde{u}^{q}$ in $\Omega$ but only its trace on $\Gamma_{m}$. One possible way around this difficulty is to normalize both $u^{q}$ and $\tilde{u}^{q}$ with respect to some norm on $\Gamma_{m}$, for instance in $L^{2}\left(\Gamma_{m}\right)$.

\section{Shape derivatives}

This section is devoted to the computation of the shape derivative of the functional $J$ defined in (3). Infinitesimal variations of an initially given shape will be considered in the same spirit as in $[10,23,26]$. Note however that the existing results focus on objective functionals which do not depend on eigenmodes. Differentiating an objective functional depending on eigenmodes is essentially new and involves an adjoint problem of a new type, to our knowledge never considered before. An approach close to ours can be found in [24], however the authors consider a fixed Hilbert space since they work on a fixed domain $\Omega$ (the varying parameters control material properties). On the other hand, in [24] no objective functional is considered (only the derivatives of the eigenmodes are computed) so no adjoint state is involved.

\subsection{The adjoint state}

We recall some basic notions on the adjoint method as introduced by Cea in [9]. Given a functional $J(\theta)$ to minimize/maximize, the goal is to find a control $\theta$ producing the state $u_{\theta}$ that minimizes/maximizes the functional. Namely, we consider a functional of the form

$$
J(\theta)=\mathcal{J}\left(\theta, u_{\theta}\right),
$$

which depends on $\theta$ in a direct manner and also through the solution $u_{\theta}$ of a state equation which may be a partial differential equation having the variational formulation

$$
A_{\theta}\left(u_{\theta}, v\right)=l_{\theta}(v), \forall v \in H .
$$

In the above, $A_{\theta}$ is a bilinear, continuous and coercive application depending on a parameter $\theta, l_{\theta}$ is a linear, continuous application depending on $\theta, H$ is a fixed Hilbert space.

The physical quantity $\theta$ is called control and one is looking for a $\theta$ which minimizes/maximizes $J$. Since a necessary condition for extremum is the vanishing of the total derivative

$$
\frac{d J}{d \theta}=0
$$

one of the most important ingredients is the analytic calculus of the above referred derivative. The state $u_{\theta}$ depends on $\theta$ in an implicit way, through the problem (4) and one seeks to describe the variation of $u_{\theta}$ in terms of infinitesimal variations 
of $\theta$, under convenient differentiability hypothesis on the families of operators $A_{\theta}$ and $l_{\theta}$ with respect to $\theta$. Note that the total derivative of $J$ involves the derivative of $u_{\theta}$ with respect to $\theta$ :

$$
\frac{d J}{d \theta}(\theta) \tau=\frac{\partial \mathcal{J}}{\partial \theta}\left(\theta, u_{\theta}\right) \tau+\frac{\partial \mathcal{J}}{\partial u}\left(\theta, u_{\theta}\right) \frac{d u_{\theta}}{d \theta}(\theta) \tau .
$$

In the above formula (5) and for the whole present section $\tau$ stays for an infinitesimal variation of $\theta$. The difficulty lays in the fact that the term $\frac{\partial \mathcal{J}}{\partial u}\left(\theta, u_{\theta}\right) \frac{d u_{\theta}}{d \theta}(\theta) \tau$ involves $\frac{d u_{\theta}}{d \theta}(\theta) \tau$; recall that $u_{\theta}$ depends on $\theta$ implicitly through problem (4). It is the adjoint method that allows one to transform the implicit dependency of $\frac{\partial \mathcal{J}}{\partial u}\left(\theta, u_{\theta}\right) \frac{d u_{\theta}}{d \theta}(\theta) \tau$ into an explicit one with respect to $\tau$.

The adjoint problem is introduced as

$$
A_{\theta}\left(p_{\theta}, w\right)=\frac{\partial \mathcal{J}}{\partial u}\left(\theta, u_{\theta}\right) w \forall w \in H,
$$

its solution $p_{\theta} \in H$ is called the adjoint state. Then under symmetry hypothesis on the operators $A_{\theta}$ it is possible to prove that the implicit term in the expression of (5) has the form:

$$
\frac{\partial \mathcal{J}}{\partial u}\left(\theta, u_{\theta}\right) \frac{d u_{\theta}}{d \theta}(\theta) \tau=A_{\theta}\left(p_{\theta}, \frac{d u_{\theta}}{d \theta}(\theta) \tau\right)=A_{\theta}\left(\frac{d u_{\theta}}{d \theta}(\theta) \tau, p_{\theta}\right)=\left(-\frac{d A_{\theta}}{d \theta}\left(u_{\theta}, p_{\theta}\right)+\frac{d l_{\theta}}{d \theta}\left(p_{\theta}\right)\right) \tau .
$$

Therefore, the total derivative of $J$ writes as the following expression, where the dependency on $\tau$ is now explicit:

$$
\frac{d J}{d \theta}(\theta) \tau=\left(\frac{\partial \mathcal{J}}{\partial \theta}\left(\theta, u_{\theta}\right)-\frac{d A_{\theta}}{d \theta}\left(u_{\theta}, p_{\theta}\right)+\frac{d l_{\theta}}{d \theta}\left(p_{\theta}\right)\right) \tau .
$$

\subsection{Domain variation for shape optimization}

We are interested in a setting where the parameter $\theta$ controls the shape of the domain $\Omega \backslash V$, thus the space $H$ itself varies with the parameter $\theta$ and the standard procedure sketched above in subsection 3.1 must be adapted. In the following we recall the classical approach of Hadamard [13] of varying the shape of a domain, following the description in [19].

Denote by $I+\theta$ the diffeomorphism that models the variation of the domain in the sense that $\Omega \backslash V=(I+\theta)\left(\Omega \backslash V_{0}\right)$. We shall denote by $\mathcal{U}_{a d}$ the set of admissible domains $\Omega \backslash V$, that is all domains that verify the above relation for some diffeomorphism $I+\theta$.

If $\theta \in W^{1, \infty}\left(\mathbb{R}^{d} ; \mathbb{R}^{d}\right)$ verifies

$$
\|\theta\|_{W^{1, \infty}\left(\mathbb{R}^{d} ; \mathbb{R}^{d}\right)}<1,
$$

then the function $I+\theta$ is invertible and belongs to the space

$$
\left\{T \in W_{\text {loc }}^{1, \infty}\left(\mathbb{R}^{d} ; \mathbb{R}^{d}\right) \text { such that } T^{-1} \in W_{\text {loc }}^{1, \infty}\left(\mathbb{R}^{d} ; \mathbb{R}^{d}\right)\right\} .
$$

Given $J: \mathcal{U}_{a d} \rightarrow \mathbb{R}, J$ is called differentiable with respect to the domain, in $\Omega \backslash V_{0}$, if the application

$$
\theta \mapsto J\left((I+\theta)\left(\Omega \backslash V_{0}\right)\right)
$$

is Fréchet differentiable in $\theta=0$.

The approach described in [19] consists in performing a change of variables in the integral operators $A_{\theta}$ and $l_{\theta}$, thus transporting the state equation (4) from the varying domain $\Omega \backslash V$ to the fixed domain $\Omega \backslash V_{0}$. This allows one to use the results in subsection 3.1 and obtain an adjoint problem identical to (6). We do not present more details here; the proof of Theorem 1 follows the same idea.

\subsection{Main result}

Unlike the approaches described above, the present paper is concerned with an eigenvalue problem (1) which is essentially different from the elliptic problem (4). The unknowns in the variational formulation (2) are $\Lambda$ and $u$, thus on the right hand side of the equation a trilinear form appears. Moreover, the objective functional in (3) depends not only on the eigenvalues $\Lambda^{q}$ but also on the eigenmodes $u^{q}$. Thus, the computation of the adjoint state and of the shape derivative must be carried out from scratch. Actually, it turns out that several specific adjoint problems must be introduced, as many as the number of eigenmodes on which the functional depends.

Theorem 1. Suppose the first eigenvalues $\Lambda^{q}, 1 \leq q \leq N$, are simple. Then, the shape derivative of $J$, that is, the Fréchet derivative of the application given in (7), is equal to 


$$
\begin{aligned}
& J^{\prime}(\theta=0)(\tau)=2 \sum_{q=1}^{N}\left(\Lambda^{q}-\tilde{\Lambda}^{q}\right) \frac{d \Lambda^{q}}{d \theta}(\tau) \\
& +\sum_{q=1}^{N}\left[\int_{\Omega \backslash V_{0}} \rho\left(u^{q}\right)_{i}\left(p^{q}\right)_{i} d x_{0}\left(\frac{d \Lambda^{q}}{d \theta}(\tau)+\int_{\partial V_{0}} \rho\left(u^{q}\right)_{i}^{2} \tau_{\alpha} n_{\alpha} d s\right)-\right. \\
& \left.-\int_{\partial V_{0}}\left[C_{i j k l}\left(p^{q}\right)_{k, l}\left(u^{q}\right)_{i, j}-\Lambda^{q} \rho\left(u^{q}\right)_{i}\left(p^{q}\right)_{i}\right] \tau_{\alpha} n_{\alpha} d s\right]
\end{aligned}
$$

where

$$
\frac{d \Lambda^{q}}{d \theta}(\tau)=\int_{\partial V_{0}}\left[C_{i j k l}\left(u^{q}\right)_{k, l}\left(u^{q}\right)_{i, j}-\Lambda^{q} \rho\left(u^{q}\right)_{i}^{2}\right] \tau_{\alpha} n_{\alpha} d s
$$

and for each index $q, 1 \leq q \leq N, p^{q}$ is the adjoint state associated to the eigensolution $\left(\Lambda^{q}, u^{q}\right)$. The adjoint state $p^{q}$ is the solution of the adjoint problem below, for all $w \in\left(H^{1}\left(\Omega \backslash V_{0}\right)\right)^{2}$ satisfying the homogeneous Dirichlet condition on $\Gamma_{D}$,

$$
\begin{aligned}
& \int_{\Omega \backslash V_{0}}\left[C_{i j k l}\left(p^{q}\right)_{k, l} w_{i, j}-\Lambda^{q} \rho\left(p^{q}\right)_{i} w_{i}\right] d x_{0}-2 \int_{\Omega \backslash V_{0}} \rho\left(u^{q}\right)_{i}\left(p^{q}\right)_{i} d x_{0} \int_{\Omega \backslash V_{0}} \rho\left(u^{q}\right)_{i} w_{i} d x_{0}= \\
= & \int_{\Gamma_{m}}\left(\left(u^{q}\right)_{i}-\left(\tilde{u}^{q}\right)_{i}\right) w_{i} d s,
\end{aligned}
$$

which in classical formulation writes:

$$
\left\{\begin{array}{l}
\nabla \cdot\left(C \epsilon\left(p^{q}\right)\right)+\Lambda^{q} \rho p^{q}+2\left(\int_{\Omega \backslash V_{0}} \rho u^{q} p^{q} d x_{0}\right) \rho u^{q}=0 \quad \text { in } \Omega \backslash V_{0}, \\
p^{q}=0 \text { on } \Gamma_{D}, \\
\sigma\left(p^{q}\right) n=u^{q}-\tilde{u}^{q} \quad \text { on } \Gamma_{m}, \\
\sigma\left(p^{q}\right) n=0 \text { on } \partial V_{0} \cup\left(\Gamma_{N} \backslash \Gamma_{m}\right) .
\end{array}\right.
$$

Remark 4. One can write another, equivalent, formulation of (10) as follows. Denote by $\gamma^{q}$ the (known) quantity

$$
\gamma^{q}=-\frac{1}{2} \int_{\Gamma_{m}}\left(\left(u^{q}\right)_{i}-\left(\tilde{u}^{q}\right)_{i}\right)\left(u^{q}\right)_{i}
$$

Then $p^{q}$ can be computed as the solution of

$$
\left\{\begin{array}{l}
\int_{\Omega \backslash V_{0}}\left[C_{i j k l}\left(p^{q}\right)_{k, l} w_{i, j}-\Lambda^{q} \rho\left(p^{q}\right)_{i} w_{i}\right] d x_{0}=2 \gamma^{q} \int_{\Omega \backslash V_{0}} \rho\left(u^{q}\right)_{i} w_{i} d x_{0}+\int_{\Gamma_{m}}\left(\left(u^{q}\right)_{i}-\left(\tilde{u}^{q}\right)_{i}\right) w_{i} d s \\
\int_{\Omega \backslash V_{0}} \rho\left(u^{q}\right)_{i}\left(p^{q}\right)_{i} d x_{0}=\gamma^{q}
\end{array}\right.
$$

which in classical formulation writes:

$$
\left\{\begin{array}{l}
\nabla \cdot\left(C \epsilon\left(p^{q}\right)\right)+\Lambda^{q} \rho p^{q}=-2 \gamma^{q} \rho u^{q} \quad \text { in } \Omega \backslash V_{0}, \\
p^{q}=0 \text { on } \Gamma_{D}, \\
\sigma\left(p^{q}\right) n=u^{q}-\tilde{u}^{q} \quad \text { on } \Gamma_{m}, \\
\sigma\left(p^{q}\right) n=0 \text { on } \partial V_{0} \cup\left(\Gamma_{N} \backslash \Gamma_{m}\right) . \\
\int_{\Omega \backslash V_{0}} \rho\left(p^{q}\right)_{i}\left(u^{q}\right)_{i}=\gamma^{q} .
\end{array}\right.
$$


Note that, in the discretized version of (12), if we drop the last (integral) condition, we obtain a degenerate $n \times n$ system (with a singular matrix). By adding the last condition, we obtain a system with $n+1$ equations and only $n$ unknowns, having unique solution.

Remark 5. In Theorem 1 we only consider the case of simple eigenvalues. When multiple eigenvalues occur, things become more complicated. Few authors have addressed this case. The paper [12] is an example; however it does not take into account the eigenmodes. See also Section 6.5 in [25].

The following proof is somewhat formal in the sense that we do not focus on the smoothness of the boundary of the domain $\Omega \backslash V$, nor on the Sobolev spaces involved. However we think it is useful to present the computation of the shape derivative (and the link to the adjoint states) with some detail.

Proof of Theorem 1. Equation (2) depends on $\theta$ through the domain $\Omega \backslash V=(I+\theta)\left(\Omega \backslash V_{0}\right)$. It is difficult to consider infinitesimal variations in (2) because the space of functions varies with $\theta$. To overcome this problem, we shall transform the integrals over $\Omega \backslash V$ into integrals over the fixed domain $\Omega \backslash V_{0}$ and then we shall derive in $\theta$.

In order to simplify notations, we shall skip the subscript $q$ which identifies the eigenmode $\left(\Lambda^{q}, u^{q}\right)$. Denoting by $T=$ $I+\theta$ and by $S=T^{-1}=(I+\theta)^{-1}$ we define $\hat{u}=u \circ T$ hence $u=\hat{u} \circ S$. Replacing $u$ in terms of $\hat{u}$ (which is defined on the fixed domain $\Omega \backslash V_{0}$ ) in equation (2), we get

$$
\int_{\Omega \backslash V} C_{i j k l} \hat{u}_{i, \alpha} \circ S S_{\alpha, j} \hat{v}_{k, \beta} \circ S S_{\beta, l} d x=\Lambda \int_{\Omega \backslash V} \rho \hat{u}_{i} \circ S \hat{v}_{i} \circ S d x \text {. }
$$

Through the change of variable $x=T\left(x_{0}\right)$, the integrals will become all on the fixed domain $\Omega \backslash V_{0}$ :

$$
\int_{\Omega \backslash V_{0}} C_{i j k l} \hat{u}_{i, \alpha} \circ S \circ T S_{\alpha, j} \circ T \hat{v}_{k, \beta} \circ S \circ T S_{\beta, l} \circ T|\operatorname{det}(\nabla T)| d x_{0}=\Lambda \int_{\Omega \backslash V_{0}} \rho \hat{u}_{i} \circ S \circ T \hat{v}_{i} \circ S \circ T|\operatorname{det}(\nabla T)| d x_{0} .
$$

But $\operatorname{det}(\nabla T)>0$ since $T=I+\theta$ is in the neighborhood of the identity and $\operatorname{det}(I)=1$. The above equation writes then

$$
\int_{\Omega \backslash V_{0}} C_{i j k l} \hat{u}_{i, \alpha} S_{\alpha, j} \circ T \hat{v}_{k, \beta} S_{\beta, l} \circ T \operatorname{det}(\nabla T) d x_{0}=\Lambda \int_{\Omega \backslash V_{0}} \rho \hat{u}_{i} \hat{v}_{i} \operatorname{det}(\nabla T) d x_{0}
$$

and it can be derived in $\theta$. We shall compute this derivative at $\theta=0$. By denoting by $M_{i j}=S_{i, j} \circ T$, one obtains

$$
\left.\frac{d}{d \theta}\left(\int_{\Omega \backslash V_{0}} C_{i j k l} \hat{u}_{i, \alpha} M_{\alpha j} \hat{v}_{k, \beta} M_{\beta l} \operatorname{det}(\nabla T) d x_{0}\right)\right|_{\theta=0}(\tau)=\left.\frac{d}{d \theta}\left(\Lambda \int_{\Omega \backslash V_{0}} \rho \hat{u}_{i} \hat{v}_{i} \operatorname{det}(\nabla T) d x_{0}\right)\right|_{\theta=0}(\tau)
$$

It may be verified that $\left.M_{i j}\right|_{\theta=0}=\delta_{i j},\left.\frac{d M_{i j}}{d \theta}\right|_{\theta=0}(\tau)=-\tau_{i, j}$ and $\left.\frac{d \operatorname{det}(\nabla T)}{d \theta}\right|_{\theta=0}(\tau)=\operatorname{div} \tau$. Note also that we chose the test function $\hat{v}$ to be fixed (independent of $\theta$ ). With the notation $\delta \hat{u}=\left.\frac{d \hat{u}}{d \theta}\right|_{\theta=0}(\tau)$ and since $\left.\hat{u}\right|_{\theta=0}=u_{0}$, the equation above writes

$$
\begin{aligned}
& \int_{\Omega \backslash V_{0}} C_{i j k l} \delta \hat{u}_{i, j} \hat{v}_{k, l} d x_{0}-\int_{\Omega \backslash V_{0}} C_{i j k l} \hat{v}_{k, l} u_{0 i, \alpha} \tau_{\alpha, j} d x_{0}- \\
& -\int_{\Omega \backslash V_{0}} C_{i j k l} u_{0 i, j} \hat{v}_{k, \beta} \tau_{\beta, l} d x_{0}+\int_{\Omega \backslash V_{0}} C_{i j k l} u_{0 i, j} \hat{v}_{k, l} \operatorname{div} \tau d x_{0}= \\
& =\left.\frac{d \Lambda}{d \theta}\right|_{\theta=0} \int_{\Omega \backslash V_{0}} \rho u_{0 i} \hat{v}_{i} d x_{0}+\Lambda \int_{\Omega \backslash V_{0}} \rho \delta \hat{u}_{i} \hat{v}_{i} d x_{0}+\Lambda \int_{\Omega \backslash V_{0}} \rho u_{0 i} \hat{v}_{i} \operatorname{div} \tau d x_{0} .
\end{aligned}
$$

We now turn to the computation of the shape derivative of the eigenvalue $\Lambda$. Since the eigenmodes are normalized in the norm $\|\cdot\|_{L_{\rho}^{2}}$, the variational formulation (2) yields the following expression for $\Lambda$ :

$$
\int_{\Omega \backslash V} C_{i j k l} u_{i, j} u_{k, l} d x=\Lambda \text {. }
$$

When transported on the fix domain $\Omega \backslash V_{0}$ one obtains 


$$
\int_{\Omega \backslash V_{0}} C_{i j k l} \hat{u}_{i, \alpha} S_{\alpha, j} \circ T \hat{u}_{k, \beta} S_{\beta, l} \circ T \operatorname{det}(\nabla T) d x_{0}=\Lambda,
$$

which by derivation in $\theta$, at $\theta=0$, gives

$$
2 \int_{\Omega \backslash V_{0}} C_{i j k l} \delta \hat{u}_{i, j} u_{0 k, l} d x_{0}-2 \int_{\Omega \backslash V_{0}} C_{i j k l} u_{0 k, l} u_{0 i, \alpha} \tau_{\alpha, j} d x_{0}+\int_{\Omega \backslash V_{0}} C_{i j k l} u_{0 i, j} u_{0 k, l} \operatorname{div} \tau d x_{0}=\left.\frac{d \Lambda}{d \theta}\right|_{\theta=0}(\tau)
$$

On the other hand, since the eigenvector $u$ is normalized, and transporting on the fixed domain $\Omega \backslash V_{0}$, one obtains

$$
\begin{aligned}
& 0=\left.\frac{d\|u\|}{d \theta}\right|_{\theta=0}=\left.\frac{d}{d \theta}\right|_{\theta=0} \int_{\Omega \backslash V} \rho u^{2} d x=\left.\frac{d}{d \theta}\right|_{\theta=0} \int_{\Omega \backslash V_{0}} \rho \hat{u}_{i}^{2} \operatorname{det}(\nabla T) d x_{0}= \\
& =2 \int_{\Omega \backslash V_{0}} \rho \delta \hat{u}_{i} u_{0 i} d x_{0}+\int_{\Omega \backslash V_{0}} \rho u_{0 i}^{2} \operatorname{div} \tau d x_{0} .
\end{aligned}
$$

Therefore

$$
2 \int_{\Omega \backslash V_{0}} \rho \delta \hat{u}_{i} u_{0 i} d x_{0}+\int_{\Omega \backslash V_{0}} \rho u_{0 i}^{2} \operatorname{div} \tau d x_{0}=0 .
$$

Writing the variational formulation (2) for the test function $v=\delta \hat{u}$, and having in mind the above relation, we get

$$
\int_{\Omega \backslash V_{0}} C_{i j k l} u_{0 k, l} \delta \hat{u}_{i, j} d x_{0}=\Lambda \int_{\Omega \backslash V_{0}} \rho u_{0 i} \delta \hat{u}_{i} d x_{0}=-\frac{1}{2} \Lambda \int_{\Omega \backslash V_{0}} \rho u_{0 i}^{2} \operatorname{div} \tau d x_{0} .
$$

After replacing the above integral in (15), the derivative of $\Lambda$ writes

$$
\left.\frac{d \Lambda}{d \theta}\right|_{\theta=0}(\tau)=-\Lambda \int_{\Omega \backslash V_{0}} \rho u_{0 i}^{2} \operatorname{div} \tau d x_{0}+\int_{\Omega \backslash V_{0}} C_{i j k l} u_{0 i, j} u_{0 k, l} \operatorname{div} \tau d x_{0}-2 \int_{\Omega \backslash V_{0}} C_{i j k l} u_{0 k, l} u_{0 i, \alpha} \tau_{\alpha, j} d x_{0} .
$$

Applying the flux-divergence theorem to each of the above terms one obtains

$$
\begin{aligned}
& \left.\frac{d \Lambda}{d \theta}\right|_{\theta=0}(\tau)=\int_{\Omega \backslash V_{0}}\left(\Lambda \rho u_{0 i}^{2}\right)_{, \alpha} \tau_{\alpha} d x_{0}-\int_{\partial \Omega \cup \partial V_{0}} \Lambda \rho u_{0 i}^{2} \tau_{\alpha} n_{\alpha} d s_{0}- \\
& -\int_{\Omega \backslash V_{0}}\left(C_{i j k l} u_{0 k, l}\right)_{, \alpha} u_{0 i, j} \tau_{\alpha} d x_{0}-\int_{\Omega \backslash V_{0}} C_{i j k l} u_{0 k, l} u_{0 i, j \alpha} \tau_{\alpha} d x_{0}+\int_{\partial \Omega \cup \partial V_{0}} C_{i j k l} u_{0 k, l} u_{0 i, j} \tau_{\alpha} n_{\alpha} d s_{0}+ \\
& +2 \int_{\Omega \backslash V_{0}}\left(C_{i j k l} u_{0 k, l}\right)_{, j} u_{0 i, \alpha} \tau_{\alpha} d x_{0}+2 \int_{\Omega \backslash V_{0}} C_{i j k l} u_{0 k, l} u_{0 i, \alpha j} \tau_{\alpha} d x_{0}-2 \int_{\partial \Omega \cup \partial V_{0}} C_{i j k l} u_{0 k, l} u_{0 i, \alpha} \tau_{\alpha} n_{j} d s_{0}
\end{aligned}
$$

In the first parcel of the above expression we differentiate: $\left(\Lambda \rho u_{0 i}^{2}\right)_{, \alpha}=2 \Lambda \rho u_{0 i} u_{0 i, \alpha}$ and, due to the classical formulation of the eigenvalue problem (1), the first integral cancels with the sixth integral. Also the third, fourth and seventh integrals sum up to zero. On the other hand, due to the homogeneous Neumann condition $C_{i j k l} u_{0 k, l} n_{j}=0$ on both $\Gamma_{N}$ and $\partial V_{0}$, in the last parcel the domain of integration can be replaced by $\Gamma_{D}$. So, only the boundary terms remain in the expression of the derivative of $\Lambda$ :

$$
\left.\frac{d \Lambda}{d \theta}\right|_{\theta=0} ^{(\tau)=} \int_{\partial \Omega \cup \partial V_{0}}\left(C_{i j k l} u_{0 k, l} u_{0 i, j}-\Lambda \rho u_{0 i}^{2}\right) \tau_{\alpha} n_{\alpha} d s_{0}-2 \int_{\Gamma_{D}} C_{i j k l} u_{0 k, l} u_{0 i, \alpha} \tau_{\alpha} n_{j} d s_{0}
$$

Since on $\Gamma_{D}$ one has $u_{i}=0, \nabla u$ is parallel to the normal $n$ and therefore $\nabla u_{i}=g_{i} n$, and by components $u_{i, j}=g_{i} n_{j}$. Then $u_{i, j} n_{j}=g_{i} n_{j} n_{j}=g_{i}$. Consequently $u_{i, j}=u_{i, \alpha} n_{\alpha} n_{j}$. Hence $C_{i j k l} u_{k, l} u_{i, \alpha} \tau_{\alpha} n_{j}=C_{i j k l} u_{k, l} u_{i, \beta} n_{\beta} n_{\alpha} \tau_{\alpha} n_{j}=$ $C_{i j k l} u_{k, l} u_{i, \beta} n_{\beta} n_{j} \tau_{\alpha} n_{\alpha}=C_{i j k l} u_{k, l} u_{i, j} \tau_{\alpha} n_{\alpha}$. Then the derivative of $\Lambda$ writes:

$$
\left.\frac{d \Lambda}{d \theta}\right|_{\theta=0}(\tau)=\int_{\Gamma_{N} \cup \partial V_{0}}\left(C_{i j k l} u_{0 k, l} u_{0 i, j}-\Lambda \rho u_{0 i}^{2}\right) \tau_{\alpha} n_{\alpha} d s_{0}-\int_{\Gamma_{D}}\left(C_{i j k l} u_{0 k, l} u_{0 i, j}+\Lambda \rho u_{0 i}^{2}\right) \tau_{\alpha} n_{\alpha} d s_{0},
$$

and since in the context under consideration the only moving boundary is $\partial V$, one obtains the formula (9) in the theorem. 
Going back to (14), the problem that defines the derivative of the eigenmode $u_{0}$, writes:

$$
\begin{aligned}
& \int_{\Omega \backslash V_{0}} C_{i j k l} \delta \hat{u}_{i, j} \hat{v}_{k, l} d x_{0}-\Lambda \int_{\Omega \backslash V_{0}} \rho \delta \hat{u}_{i} \hat{v}_{i} d x_{0}=\int_{\Omega \backslash V_{0}} C_{i j k l} \hat{v}_{k, l} u_{0 i, \alpha} \tau_{\alpha, j} d x_{0}+\int_{\Omega \backslash V_{0}} C_{i j k l} u_{0 i, j} \hat{v}_{k, \alpha} \tau_{\alpha, l} d x_{0}+ \\
& +\left.\frac{d \Lambda}{d \theta}\right|_{\theta=0}(\tau) \int_{\Omega \backslash V_{0}} \rho u_{0 i} \hat{v}_{i} d x_{0}+\int_{\Omega \backslash V_{0}}\left(-C_{i j k l} u_{0 i, j} \hat{v}_{k, l}+\Lambda \rho u_{0 i} \hat{v}_{i}\right) \operatorname{div} \tau d x_{0} .
\end{aligned}
$$

However, for $\hat{v}=u_{0}$, the righthand and the lefthand terms of the above variational equation are identically equal to zero, therefore the supplementary condition (16) is to be taken into account. For a general test function $\hat{v}$ one may write the supplementary condition (16) as

$$
-\int_{\Omega \backslash V_{0}} \rho u_{0 i} \hat{v}_{i} d x_{0} \int_{\Omega \backslash V_{0}} \rho \delta \hat{u}_{i} u_{0 i} d x_{0}=\frac{1}{2} \int_{\Omega \backslash V_{0}} \rho u_{0 i} \hat{v}_{i} d x_{0} \int_{\Omega \backslash V_{0}} \rho u_{0 i} u_{0 i} \operatorname{div} \tau d x_{0} .
$$

The above condition is significant precisely on the space generated by the eigenvector $u_{0}$, where the variational formulation (18) gives no information. On the other hand, on the orthogonal complement of $u_{0}$, this information is irrelevant and the variational formulation (18) is substantial. A linear combination between (18) and (19) produces the following concise variational formulation which defines the derivative of the eigenmode $u$ as its unique solution

$$
\begin{aligned}
& \int_{\Omega \backslash V_{0}} C_{i j k l} \delta \hat{u}_{i, j} \hat{v}_{k, l} d x_{0}-\Lambda \int_{\Omega \backslash V_{0}} \rho \delta \hat{u}_{i} \hat{v}_{i} d x_{0}-\eta \int_{\Omega \backslash V_{0}} \rho u_{0 i} \hat{v}_{i} d x_{0} \int_{\Omega \backslash V_{0}} \rho \delta \hat{u}_{i} u_{0 i} d x_{0}= \\
& =\int_{\Omega \backslash V_{0}} C_{i j k l} \hat{v}_{k, l} u_{0 i, \alpha} \tau_{\alpha, j} d x_{0}+\int_{\Omega \backslash V_{0}} C_{i j k l} u_{0 i, j} \hat{v}_{k, \alpha} \tau_{\alpha, l} d x_{0}+ \\
& +\left.\frac{d \Lambda}{d \theta}\right|_{\theta=0} ^{(\tau)} \int_{\Omega \backslash V_{0}} \rho u_{0 i} \hat{v}_{i} d x_{0}+\int_{\Omega \backslash V_{0}}\left(-C_{i j k l} u_{0 i, j} \hat{v}_{k, l}+\Lambda \rho u_{0 i} \hat{v}_{i}\right) \operatorname{div} \tau d x_{0}+ \\
& +\frac{\eta}{2} \int_{\Omega \backslash V_{0}} \rho u_{0 i} \hat{v}_{i} d x_{0} \int_{\Omega \backslash V_{0}} \rho u_{0 i} u_{0 i} \operatorname{div} \tau d x_{0},
\end{aligned}
$$

where $\eta$ is an arbitrary real coefficient to be chosen later.

From now on, we shall use the superscript $q$ to distinguish between different eigenvalues and eigenmodes. For each $q$ between 1 and $N$, consider the adjoint problem in the form:

$$
\begin{aligned}
& \int_{\Omega \backslash V_{0}}\left[C_{i j k l}\left(p^{q}\right)_{k, l} w_{i, j}-\Lambda^{q} \rho\left(p^{q}\right)_{i} w_{i}\right] d x_{0}-\eta \int_{\Omega \backslash V_{0}} \rho\left(u_{0}^{q}\right)_{i}\left(p^{q}\right)_{i} d x_{0} \int_{\Omega \backslash V_{0}} \rho\left(u_{0}^{q}\right)_{i} w_{i} d x_{0}= \\
= & 2 \int_{\Gamma_{m}}\left(\left(u_{0}^{q}\right)_{i}-\left(\tilde{u}^{q}\right)_{i}\right) w_{i} d s
\end{aligned}
$$

The left hand side of the adjoint problem has the same structure as the bilinear form in (20) while the right hand side is equal to $\frac{\partial J}{\partial u^{q}} w$. Then the total derivative of the functional $J$ writes:

$$
J^{\prime}(\theta=0)(\tau)=\left.\frac{d J}{d \theta}\right|_{\theta=0}(\tau)=\left.\frac{\partial J}{\partial \theta}\right|_{\theta=0} ^{(\tau)}+\left.\sum_{q=1}^{N} \frac{\partial J}{\partial \Lambda^{q}} \frac{d \Lambda^{q}}{d \theta}\right|_{\theta=0}(\tau)+\sum_{q=1}^{N} \frac{\partial J}{\partial u^{q}} \delta \hat{u}^{q}
$$

Due to the adjoint problem (21) for $w=\delta \hat{u}^{q}$ the second term in the above expression has the form

$$
\int_{\Omega \backslash V_{0}}\left[C_{i j k l}\left(p^{q}\right)_{k, l} w_{i, j}-\Lambda^{q} \rho\left(p^{q}\right)_{i} w_{i}\right] d x_{0}-\eta \int_{\Omega \backslash V_{0}} \rho\left(u_{0}^{q}\right)_{i}\left(p^{q}\right)_{i} d x_{0} \int_{\Omega \backslash V_{0}} \rho\left(u_{0}^{q}\right)_{i} w_{i} d x_{0}
$$

and having in mind problem (20) for the test function $\hat{v}=p^{q}$, it is equal to 


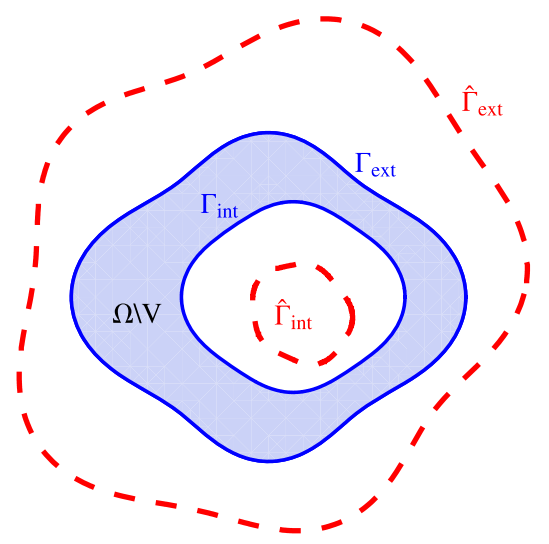

Fig. 2. The boundaries $\Gamma_{\text {int }}$ and $\Gamma_{\text {ext }}$ and the artificial boundaries $\hat{\Gamma}_{\text {int }}$ and $\hat{\Gamma}_{e x t}$.

$$
\begin{aligned}
& =\int_{\Omega \backslash V_{0}} C_{i j k l}\left(p^{q}\right)_{k, l}\left(u_{0}^{q}\right)_{i, \alpha} \tau_{\alpha, j} d x_{0}+\int_{\Omega \backslash V_{0}} C_{i j k l}\left(u_{0}^{q}\right)_{i, j}\left(p^{q}\right)_{k, \alpha} \tau_{\alpha, l} d x_{0}+\left.\frac{d \Lambda}{d \theta}\right|_{\theta=0}(\tau) \int_{\Omega \backslash V_{0}} \rho\left(u_{0}^{q}\right)_{i}\left(p^{q}\right)_{i} d x_{0} \\
& +\int_{\Omega \backslash V_{0}}\left(-C_{i j k l}\left(u_{0}^{q}\right)_{i, j}\left(p^{q}\right)_{k, l}+\Lambda \rho\left(u_{0}^{q}\right)_{i}\left(p^{q}\right)_{i}\right) d i v \tau d x_{0}+\frac{\eta}{2} \int_{\Omega \backslash V_{0}} \rho\left(u_{0}^{q}\right)_{i}\left(p^{q}\right)_{i} d x_{0} \int_{\Omega \backslash V_{0}} \rho\left(u_{0}^{q}\right)_{i}\left(u_{0}^{q}\right)_{i} d i v \tau d x_{0}
\end{aligned}
$$

Applying the flux-divergence theorem to each volume integral and after canceling terms, we see that it is equal to

$$
\begin{aligned}
& =\int_{\Omega \backslash V_{0}} \rho\left(u_{0}^{q}\right)_{i}\left(p^{q}\right)_{i} d x_{0}\left(\frac{d \Lambda}{d \theta}(\tau)+\frac{\eta}{2} \int_{\Gamma_{N} \cup \partial V_{0}} \rho\left(u_{0}^{q}\right)_{i}^{2} \tau_{\alpha} n_{\alpha} d s\right)- \\
& +\int_{\Gamma_{D}} C_{i j k l}\left(p^{q}\right)_{k, l}\left(u_{0}^{q}\right)_{i, j} \tau_{\alpha} n_{\alpha} d s-\int_{\Gamma_{N} \cup \partial V_{0}}\left[C_{i j k l}\left(p^{q}\right)_{k, l}\left(u_{0}^{q}\right)_{i, j}-\Lambda \rho\left(u_{0}^{q}\right)_{i}\left(p^{q}\right)_{i}\right] \tau_{\alpha} n_{\alpha} d s
\end{aligned}
$$

Any non-zero value of $\eta$ will do, but we shall choose $\eta=2$ for convenience. Since $\Gamma_{N}$ and $\Gamma_{D}$ do not vary, and having in mind the formula (9) of the derivative of $\Lambda$, it turns out that the derivative of $J$ is given by (8).

Remark 6. It is straightforward to generalize the above computations for computing the derivative of more general functionals depending on the eigenvalues and on the eigenmodes. For this, it suffices to replace the right hand side of the variational form of the adjoint problem (10) by the corresponding expression of $\frac{\partial J}{\partial u^{q}} \delta \hat{u}^{q}$.

\section{Numerical methods}

Given the Lamé coefficients $\lambda, \mu$ and the specific mass $\rho$, the fundamental tensor associated to the PDE of (1) is the Kupradze tensor

$$
\mathbb{G}_{\Lambda}=\frac{1}{\mu \kappa_{s}^{2}}\left(\kappa_{s}^{2} G_{s}(x) \mathbb{I}+\mathbb{D}\left(G_{s}-G_{p}\right)(x)\right)
$$

where $\mathbb{I}$ is the Kronecker delta, $\mathbb{D}=\partial_{i j}$ and $\kappa_{p}=\sqrt{\frac{\Lambda \rho}{\lambda+2 \mu}}, \kappa_{s}=\sqrt{\frac{\Lambda \rho}{\mu}} . G_{s}$ and $G_{p}$ are the fundamental solutions of the Helmholtz equation with frequencies $\kappa_{S}$ and $\kappa_{p}$ respectively,

$$
G_{s}(x)=\frac{i}{4} H_{0}^{(1)}\left(\kappa_{s}|x|\right), \quad G_{p}(x)=\frac{i}{4} H_{0}^{(1)}\left(\kappa_{p}|x|\right),
$$

where $H_{0}^{(1)}$ is the Hänkel function of the first kind. We will consider the numerical solution of problem (1) using the Method of Fundamental Solutions (MFS). For keeping simplicity in the exposition we will assume that $V$ is connected. The case where $V$ has several connected components can be handled in the same way. We will use the notation $\Gamma_{e x t}=\partial \Omega$, $\Gamma_{i n t}=\partial V$ and consider two artificial boundaries, $\hat{\Gamma}_{i n t}$ and $\hat{\Gamma}_{\text {ext }}$, which are the boundaries of two simply connected domains $\hat{\Omega}_{\text {int }}$ and $\hat{\Omega}_{\text {ext }}$, such that $\bar{\Omega}_{\text {int }} \subset V$ and $\bar{\Omega} \subset \hat{\Omega}_{\text {ext }}$, as illustrated in Fig. 2. We will also define $\hat{\Gamma}=\hat{\Gamma}_{i n t} \cup \hat{\Gamma}_{\text {ext }}$. The MFS approximation is built by considering shifts of the Kupradze tensor to some points placed on $\hat{\Gamma}$. 
Definition 1. An MFS approximation in the discrete set $\hat{\Gamma}_{m}=\left\{y_{1}, \ldots, y_{m}\right\} \subset \hat{\Gamma}$ is an element of the linear space $\mathcal{V}_{m}=$ $\left.\operatorname{span}\left\{\mathbb{G}_{\Lambda}\left(\bullet-y_{1}\right), \ldots, \mathbb{G}_{\Lambda}\left(\bullet-y_{m}\right)\right\}\right|_{\partial \Omega}$.

Next, we prove a density result for the MFS approximations which is an extension of a theorem proved in [4] (in the context of the Helmholtz equation and simply connected domains) to the elastic case and to domains which are not simply connected.

Theorem 2. If $\Lambda \in \mathbb{R}$ is not a Navier-Dirichlet eigenvalue of $\hat{\Omega}_{\text {int }}$, then

$$
S(\hat{\Gamma})=\operatorname{span}\left\{\left.\mathbb{G}_{\Lambda}(\cdot-y)\right|_{\Omega \backslash V}: y \in \hat{\Gamma}\right\}
$$

is dense in $\mathcal{H}_{\kappa}(\Omega \backslash V)=\left\{v \in\left(H^{1}(\Omega \backslash V)\right)^{2}: \nabla \cdot \sigma(v)+\rho \Lambda v=0\right\}$.

Proof. Take $v \in \mathcal{H}_{\kappa}(\Omega \backslash V)$, and let

$$
w(y)=\left\langle\mathbb{G}_{\Lambda}(\cdot-y), \bar{v}\right\rangle_{\left(H^{1}(\Omega \backslash V)\right)^{2} \times\left(H^{-1}(\Omega \backslash V)\right)^{2}}=\int_{\Omega \backslash V} \mathbb{G}_{\Lambda}(x-y) v(x) d x
$$

which is the Newtonian potential in $\Omega \backslash V$. We want to prove that if $w(y)=0, \forall y \in \hat{\Gamma}$, then $v \equiv 0$.

The Newtonian potential satisfies (see e.g. [18])

$$
\nabla \cdot \sigma(w)+\rho \Lambda w= \begin{cases}v, & \text { in } \Omega \backslash V \\ 0, & \text { in } \mathbb{R}^{2} \backslash(\overline{\Omega \backslash V}) .\end{cases}
$$

In particular, $\nabla \cdot \sigma(w)+\rho \Lambda w=0$ in $\mathbb{R}^{2} \backslash \hat{\Omega}_{\text {ext }}$ because $\bar{\Omega} \subset \hat{\Omega}_{\text {ext }}$. Then, since Kupradze radiation conditions are also satisfied, by the uniqueness of the exterior problem for $\Lambda \in \mathbb{R}$, we have $w=0$ in $\mathbb{R}^{2} \backslash \hat{\Omega}_{\text {ext }}$ and by analytic continuation, $w=0$ in $\mathbb{R}^{2} \backslash \Omega$. On the other hand, we have $w=0$ on $\hat{\Gamma}_{\text {int }}$ and thus $w=0$ in $\hat{\Omega}_{\text {int }}$ because $\Lambda$ is not a Navier-Dirichlet eigenvalue of $\hat{\Omega}_{i n t}$ and again by analytic continuation, $w=0$ in $V$. The Newtonian potential has no jumps, thus both in $\Gamma_{\text {ext }}$ and $\Gamma_{i n t}, w^{-}=w^{+}=0,(\sigma(w) n)^{-}=(\sigma(w) n)^{+}=0$ and we have the interior problem

$$
\left\{\begin{array}{cc}
\nabla \cdot \sigma(w)+\rho \Lambda w=v, & \Omega \backslash V \\
\sigma(w) n=w=0, & \Gamma_{\text {int }} \\
\sigma(w) n=w=0, & \Gamma_{\text {ext }} .
\end{array}\right.
$$

By Betti's formula (see e.g. [18]),

$$
\int_{\Omega \backslash V}(\bar{v} \nabla \cdot \sigma(w)-w \nabla \cdot \sigma(\bar{v})) d x=\int_{\Gamma}(\bar{v} \sigma(w) n-w \sigma(\bar{v}) n) d x=0
$$

and thus

$$
0=\int_{\Omega \backslash V}(\bar{v} \nabla \cdot \sigma(w)-w \nabla \cdot \sigma(\bar{v})) d x=\int_{\Omega \backslash V}(\bar{v}(v-\rho \Lambda w)-w(-\rho \Lambda \bar{v})) d x=\|v\|_{L^{2}(\Omega \backslash V)}^{2}
$$

which implies $v \equiv 0$.

We will calculate the eigensolutions of (1) using MFS approximations (see e.g. $[16,17,4]$ ). The displacement field $u$ is approximated by a linear combination of the form

$$
u(x) \approx \sum_{j=1}^{N^{M F S}} \mathbb{G}_{\Lambda}\left(x-y_{j}\right) a_{j}
$$

where $a_{j} \in \mathbb{C}^{2}$. In this work we follow the choice for the set of the point sources $Y=\left\{y_{j}, j=1, \ldots, N^{M F S}\right\}$ proposed in [3, 4]. By construction, the MFS approximation satisfies the PDE of the problem, since each term of the linear combination is a translation of the fundamental solution to some point source $y_{j} \notin \bar{\Omega}$. The coefficients are calculated such that the boundary conditions of the problem are approximated in some sense. We consider the sets of points

$$
X^{D}=\left\{x_{i}^{D}, i=1, \ldots, M^{D}\right\}, \quad X^{N}=\left\{x_{i}^{N}, i=1, \ldots, M^{N}\right\}, \quad X^{\partial V}=\left\{x_{i}^{\partial V}, i=1, \ldots, M^{\partial V}\right\}
$$

(almost) uniformly distributed respectively on the boundaries $\Gamma_{D}, \Gamma_{N}$ and $\partial V$, where $M^{D}+M^{N}+M^{\partial V}>N^{M F S}$. We impose the boundary conditions of the problem at these points, 


$$
u\left(x_{i}^{D}\right)=0, i=1, \ldots, M^{D}, \quad \sigma\left(u\left(x_{i}^{N}\right)\right) n=0, i=1, \ldots, M^{N}, \quad \sigma\left(u\left(x_{i}^{\partial V}\right)\right) n=0, i=1, \ldots, M^{\partial V},
$$

which can be written as a linear system of equations whose matrix depends on $\Lambda$. The approximations for the eigenvalues correspond to the values of $\Lambda$ such that we have a nonzero solution for this system and can be calculated with BetckeTrefethen subspace angle technique (cf. [8]). This technique involves the calculation of a $Q R$ factorization and the singular value decomposition of a matrix $\mathbf{A}(\boldsymbol{\Lambda})$ which depends on $\Lambda$. Then, we calculate the singular values, which are the square root of the non-zero eigenvalues of $A^{*}(\Lambda) A(\Lambda)$ and can be calculated with stable and fairly fast numerical methods (see e.g. [11]). We study the evolution of the smallest singular value as a function of $\Lambda, \sigma_{1}(\Lambda)$. The approximations for the eigenvalues are the values $\Lambda$ for which $\sigma_{1}(\Lambda) \approx 0$ (see [8] for details).

In order to solve the shape optimization problem, we need to define a class of admissible inclusions. For some $P \in \mathbb{N}$ consider the functions

$$
\gamma_{1}(t)=a_{0}^{(1)}+\sum_{j=1}^{P} a_{j}^{(1)} \cos (j t)+\sum_{j=1}^{P} b_{j}^{(1)} \sin (j t)
$$

and

$$
\gamma_{2}(t)=a_{0}^{(2)}+\sum_{j=1}^{P} a_{j}^{(2)} \cos (j t)+\sum_{j=1}^{P} b_{j}^{(2)} \sin (j t)
$$

and the vector $\mathcal{C} \in \mathbb{R}^{4 P+2}$ with all the coefficients,

$$
\mathcal{C}=\left(a_{0}^{(1)}, a_{1}^{(1)}, \ldots, a_{P}^{(1)}, b_{1}^{(1)}, \ldots, b_{P}^{(1)}, a_{0}^{(2)}, a_{1}^{(2)}, \ldots, a_{P}^{(2)}, b_{1}^{(2)}, \ldots, b_{P}^{(2)}\right) .
$$

The class of admissible inclusions is the set

$$
\mathcal{V}=\left\{V \subset \mathbb{R}^{2}: \partial V=\left\{\gamma_{1}(t), \gamma_{2}(t): t \in[0,2 \pi[\} \text { is a Jordan curve }\}\right.\right.
$$

and (for a fixed $\Omega$ ) the set of admissible domains is

$$
\mathcal{O}=\left\{\Omega \backslash\left(\bigcup_{j=1}^{N_{i}} V_{j}\right): V_{j} \text { are pairwise disjoint, } \overline{V_{j}} \subset \Omega, N_{i} \leq N_{\text {incl }}\right\},
$$

where $N_{\text {incl }}$ is the maximum of the number of inclusions. In this work we consider $N_{\text {incl }}=5$, which means that we exclude domains with more than 5 inclusions. The shape optimization of each inclusion is performed by searching for optimal vectors $\mathcal{C}$.

A drawback of this parametrization is related with the difficulty of dealing with changes of topology during the optimization procedure. To avoid these difficulties, at a first step, we use a genetic algorithm to provide a good initial guess for the BFGS algorithm. We start building a sample of 100 admissible domains generated randomly, having different numbers of inclusions $N_{i}=1,2, \ldots, N_{i n c l}=5$. Then, we calculate a few generations in the genetic algorithm. A descendant of two individuals is calculated by crossing the corresponding vectors $\mathcal{C}$. The crossing-over of two vectors $\mathcal{C}_{1}$ and $\mathcal{C}_{2}$ is defined by

$$
\mathcal{C}_{3}=t \mathcal{C}_{1}+(1-t) \mathcal{C}_{2}+\mathcal{P}
$$

where $t$ is randomly chosen in $(0,1)$ and $\mathcal{P}$ is a vector calculated randomly with the same dimension of $\mathcal{C}_{1}$ and with small norm. It is allowed to cross individuals with different number of inclusions. For instance, we can cross two domains $D_{1}, D_{2} \in \mathcal{O}$ having respectively $N_{i_{1}}$ and $N_{i_{2}}$ inclusions, for example with $N_{i_{1}}<N_{i_{2}}$. In that case, the $N_{i_{1}}$ vectors $\mathcal{C}$ defining $D_{1}$ are crossed with $N_{i_{1}}$ of the $N_{i_{2}}$ corresponding vectors of $D_{2}$. Then, we define a Bernoulli random variable $X$, with probability of success equal to 0.5 . If $X=0$, we simply exclude the remaining $N_{i_{2}}-N_{i_{1}}$ vectors $\mathcal{C}$ that were not selected to be crossed with those of the domain $D_{1}$, which means that the descendant will have just $N_{i_{1}}$ inclusions. Otherwise, if $X=1$, the $N_{i_{2}}-N_{i_{1}}$ vectors $\mathcal{C}$ of $D_{2}$ are kept unchanged in the descendant, which implies that the descendant has $N_{i_{2}}$ inclusions.

Another approach that could be considered and allows to handle changes in topology is to use a level set method (see e.g. $[1,2,12,22])$.

After calculating a good initial guess by the genetic algorithm, we apply the BFGS algorithm to minimize the functional $J$. Note that by the definition of $J$, we need to calculate $\left\|\left.u^{q}\right|_{\Gamma_{m}}-\left.\tilde{u}^{q}\right|_{\Gamma_{m}}\right\|_{L^{2}\left(\Gamma_{m}\right)}^{2}$, for $q=1, \ldots, N$.

Remark 7. There is an ambiguity because $u^{q}$ and $-u^{q}$ are both normalized eigenfunctions. To circumvent this problem and decide the 'correct' sign in the eigenfunction, for each $q=1, \ldots, N$, if

$$
\left\|-\left.u^{q}\right|_{\Gamma_{m}}-\left.\tilde{u}^{q}\right|_{\Gamma_{m}}\right\|_{L^{2}\left(\Gamma_{m}\right)}^{2}<\left\|\left.u^{q}\right|_{\Gamma_{m}}-\left.\tilde{u}^{q}\right|_{\Gamma_{m}}\right\|_{L^{2}\left(\Gamma_{m}\right)}^{2},
$$

we change the sign of the eigenfunction and $u^{q}$ is replaced by $-u^{q}$. Another possibility would be to use in the cost function, instead of the $L^{2}$ distance between $u_{q}$ and $\tilde{u}_{q}$, the quantity $-\left(u_{q}, \tilde{u}_{q}\right)_{L^{2}\left(\Gamma_{m}\right)}^{2}$, where 


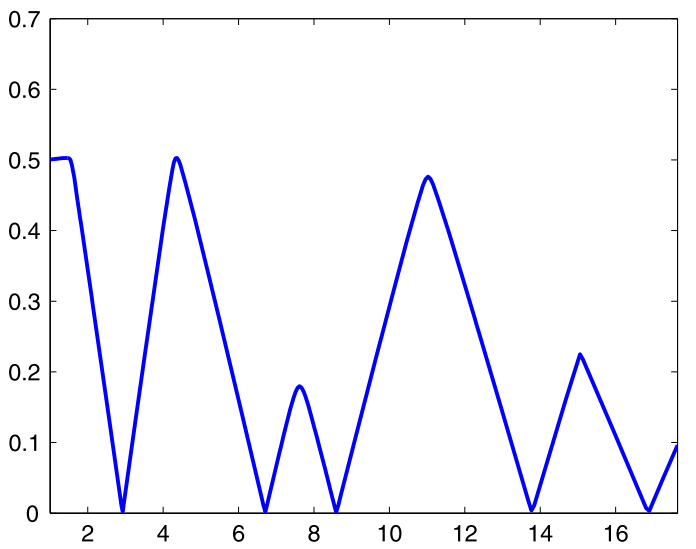

Fig. 3. $\sigma_{1}(\Lambda)$ for $\Lambda \in[1,17.5]$.
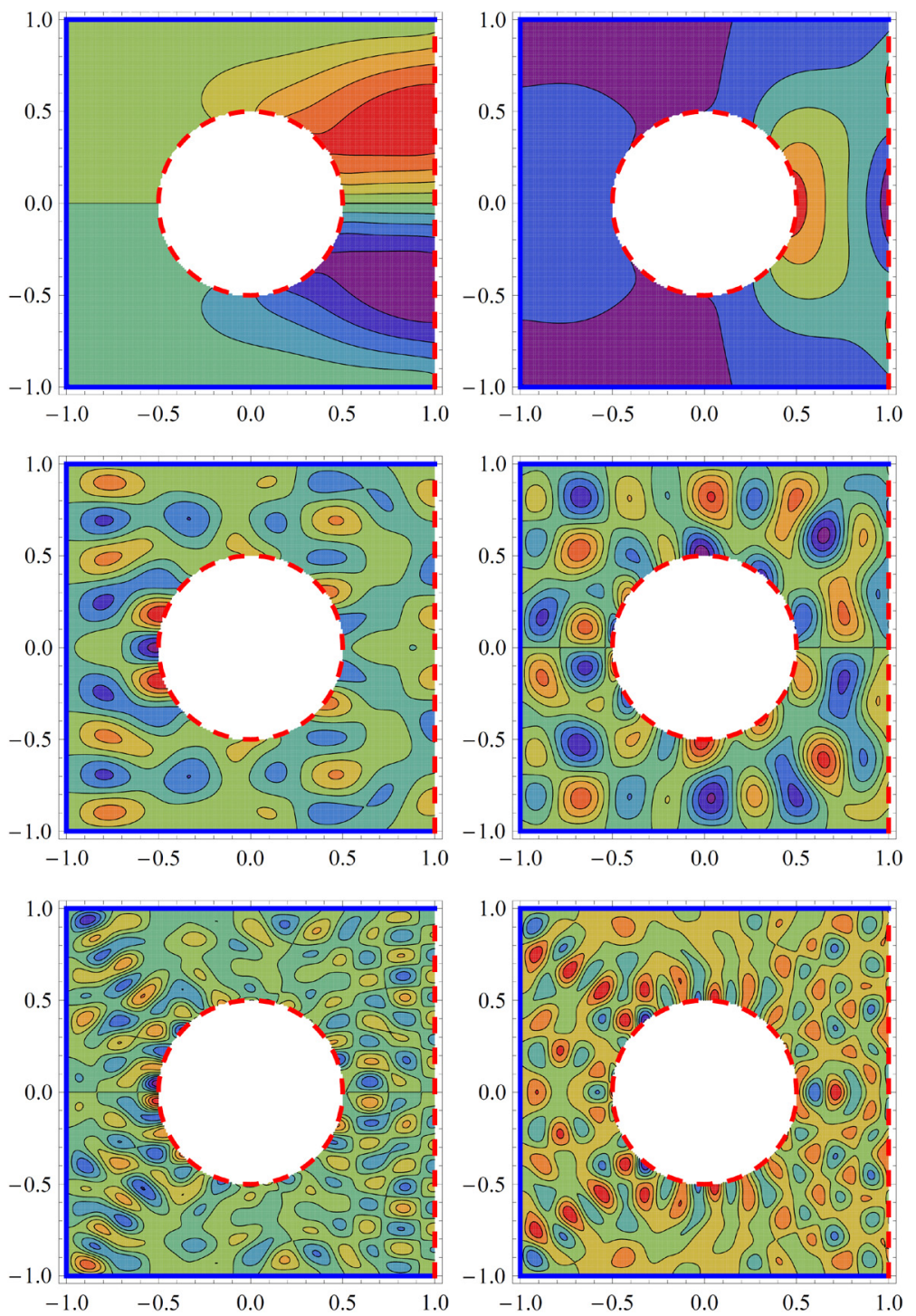

Fig. 4. First components (left plots) and second components (right plots) of the eigenfunction associated to $\Lambda_{1} \approx 2.91741, \Lambda_{100} \approx 289.41906$ and $\Lambda_{600} \approx$ 1066.68372 . 


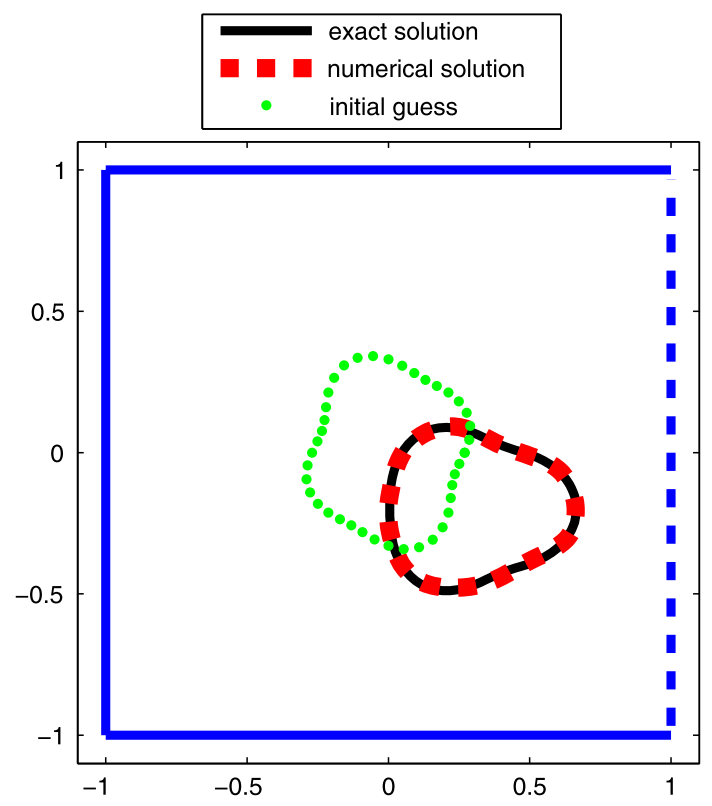

Fig. 5. The target hole $\tilde{V}$ (continuous black line), the initial guess (green dots) and the computed hole $V$ (red dashed line). (For interpretation of the colors in this figure, the reader is referred to the web version of this article.)

$$
(v, w)_{L^{2}\left(\Gamma_{m}\right)}^{2}=\int_{\Gamma_{m}} v \cdot w d s .
$$

By minimizing this quantity one forces the function $\left.u^{q}\right|_{\Gamma_{m}}$ to become colinear with $\left.\tilde{u}^{q}\right|_{\Gamma_{m}}$.

The shape gradient is calculated using Theorem 1. The adjoint problem (13) is solved using a Kansa-type method of fundamental solutions (cf. [5]) which allows for the numerical solution of boundary value problems with inhomogeneous PDE's in the framework of the MFS, but with several test frequencies.

\section{Numerical results}

In this section we present some numerical results illustrating the good performance of the numerical method. Before going to the problem of determining the inclusion $V$, we illustrate the application of the MFS for solving the direct problem (1). We consider $\Omega=[-1,1] \times[-1,1], V$ is a ball with radius equal to 0.5 , centered at the origin and $\Gamma_{N}=\{1\} \times[-1,1]$, $\Gamma_{D}=\partial \Omega \backslash \Gamma_{N}$. In this example we take $M_{D}=150, M_{N}=50, M_{\partial V}=110$ and $N^{M F S}=200$ (80 points on $\hat{\Gamma}_{i n t}$ and 120 points on $\hat{\Gamma}_{\text {ext }}$ ). In Fig. 3 we plot the smallest singular value, $\sigma_{1}(\Lambda)$, obtained with Betcke-Trefethen subspace angle technique, for $\Lambda \in[1,17.5]$ which allows to locate 5 eigenvalues in this interval $\left(\Lambda_{1} \approx 2.91741, \Lambda_{2} \approx 6.70115, \Lambda_{3} \approx 8.59045, \Lambda_{4} \approx\right.$ $13.77762, \Lambda_{5} \approx 16.86712$ ). It is well known that the MFS can be highly accurate, even for high frequencies (cf. [7]), and this is clearly an advantage when compared with classical mesh-type methods such as the Finite Element Method which would need a huge mesh to deal with the oscillatory behavior of the solution. In Fig. 4 we plot the first and second components of eigenfunctions associated to three eigenvalues, $\Lambda_{1} \approx 2.91741$ and higher eigenvalues $\Lambda_{100} \approx 289.41906$ and $\Lambda_{600} \approx 1066.68372$.

Next we show some numerical results for the solution of the inverse problem of determining the hole $\tilde{V}$ and will denote by $V$ the current hole, approximating the target hole $\tilde{V}$. In all the experiments we take $\lambda=\mu=1$ and $\rho \equiv 1$. We always assume that the eigenvalues are simple, due to the inherent limitation in Theorem 1.

In the first example we consider just one connected hole. Again, we consider $\Omega=[-1,1] \times[-1,1], \Gamma_{N}=\{1\} \times[-1,1]$, $\Gamma_{D}=\partial \Omega \backslash \Gamma_{N}, M_{D}=150, M_{N}=50, M_{\partial V}=110$ and $N^{M F S}=200$ (80 points on $\hat{\Gamma}_{\text {int }}$ and 120 points on $\hat{\Gamma}_{\text {ext }}$ ). In Fig. 5 we plot $\partial \Omega$ in blue, $\Gamma_{D}$ is plotted with a continuous line, while $\Gamma_{N}$ is marked with a red dashed line. We plot $\partial \tilde{V}$, the boundary of the target hole, with a continuous black line. The boundary of the initial guess that was obtained from the genetic algorithm is marked with green dots and the numerical solution obtained using the BFGS algorithm is plotted with a dashed red line. We can observe that we can determine the location and shape of the hole with accuracy. In this simulation, we consider $N=5$ and obtain $J(V)=9.7 \times 10^{-5}$. We performed several tests using different values of $N$ and were able to obtain also very good reconstructions of $\tilde{V}$, even with $N=1$. Next, we illustrate the convergence in this example. In Fig. 6 we plot the evolution of the functional $J$ during the optimization procedure. The results for $n=0,1,2,3$ correspond to evaluations of the functional at the initial sample of domains and three generations of the genetic algorithm. Then, we show the results for the BFGS iterations using the 'best' domain from the last generation of the genetic algorithm as initial guess. 


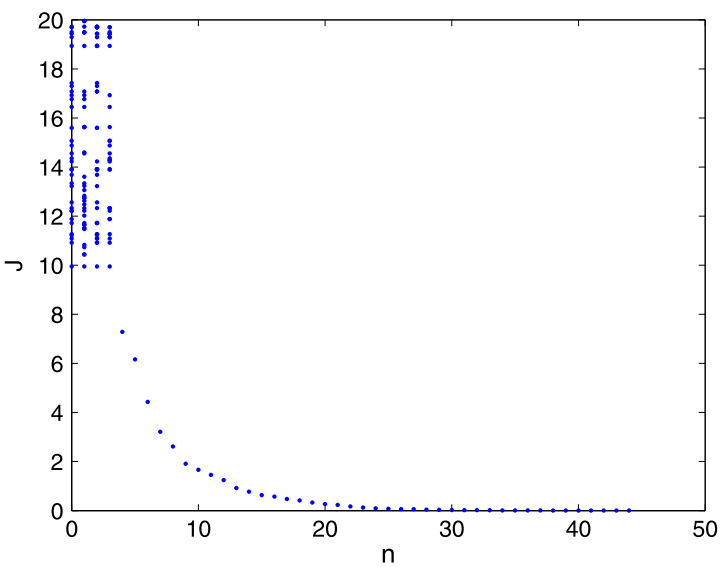

Fig. 6. Convergence results - evolution of the functional $J$ during the optimization procedure.

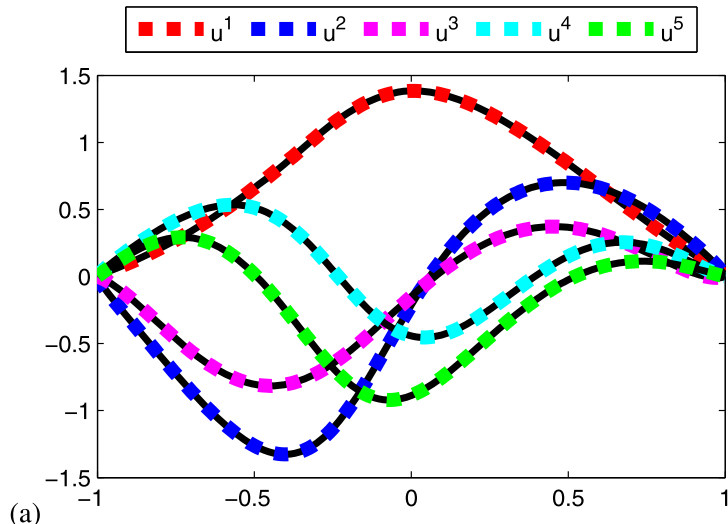

(a)

Fig. 7. (a) First components of the target $\left.\tilde{u}^{q}\right|_{\Gamma_{N}}$ (continuous black line) and $\left.u^{q}\right|_{\Gamma_{N}}$ (dashed lines), for $q=1, \ldots, 5$; (b) similar results for the second component of the eigenfunctions.

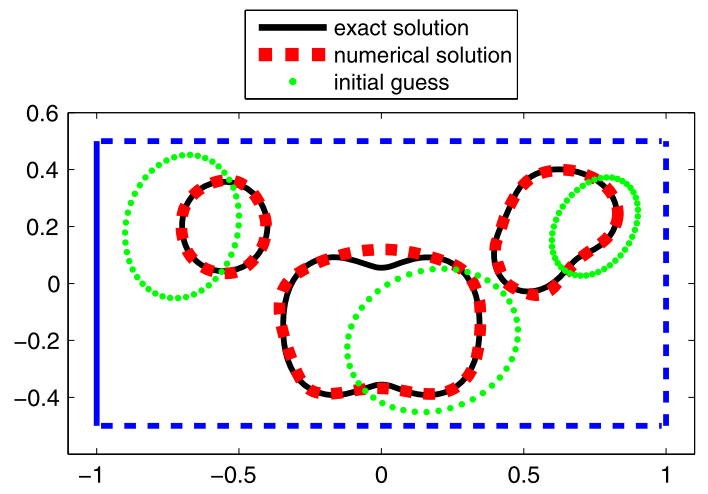

Fig. 8. The target hole $\tilde{V}$ (continuous black line), the initial guess (green dots) and the computed hole $V$ (red dashed line). (For interpretation of the colors in this figure, the reader is referred to the web version of this article.)

In Fig. 7-left we plot the traces on $\Gamma_{N}$ of the first components of the first five eigenfunctions of the exact solution (corresponding to the target hole) with a continuous black line. The numerical approximations for these quantities are marked with dashed lines. In Fig. 7-right we plot similar results for the second components of the eigenfunctions.

In the second example we consider a cantilever with three target holes. In this case, $\Omega=[-1,1] \times[-0.5,0.5], \Gamma_{D}=$ $\{-1\} \times[-0.5,0.5], \Gamma_{N}=\Gamma_{1} \cup \Gamma_{2} \cup \Gamma_{3}$, where $\Gamma_{1}=\{1\} \times[-0.5,0.5], \Gamma_{2}=[-1,1] \times\{0.5\}$ and $\Gamma_{3}=[-1,1] \times\{0.5\}$. We take $M_{D}=50, M_{N}=150, M_{\partial V}=300$ (100 collocation points on each of the boundaries of the three holes) and $N^{M F S}=500$ (100 points on each of the three auxiliary curves and 200 points on $\hat{\Gamma}_{\text {ext }}$ ). In Fig. 8 we plot with continuous black line the boundaries of the three components of the target hole $\tilde{V}$, with green dots the initial guess and with dashed red lines the 


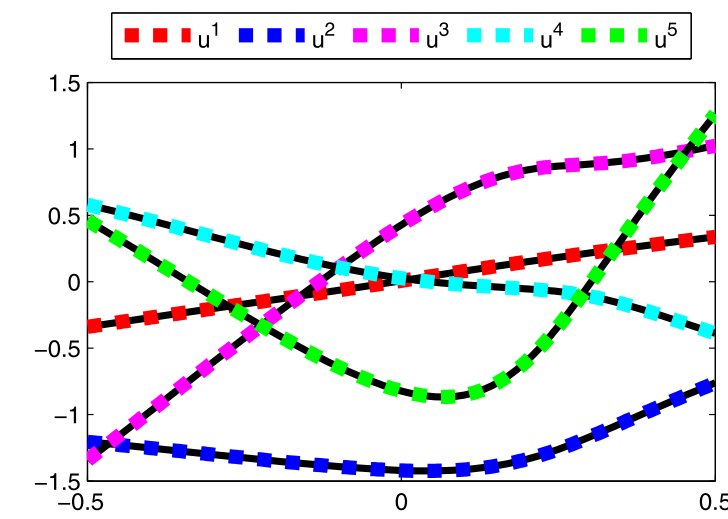

(a)

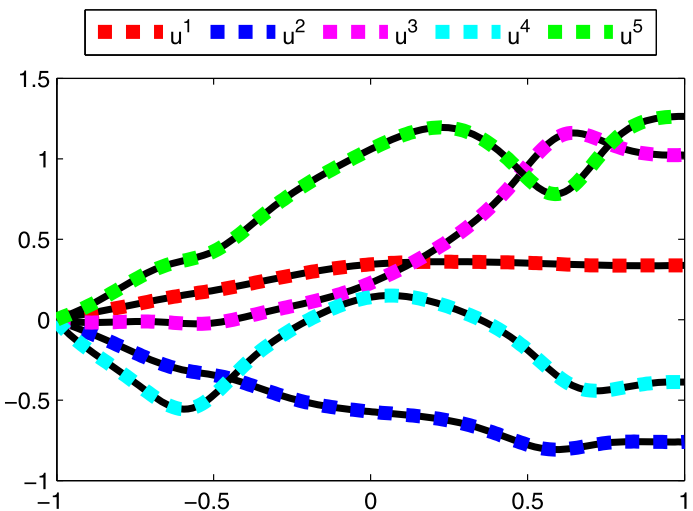

(b)

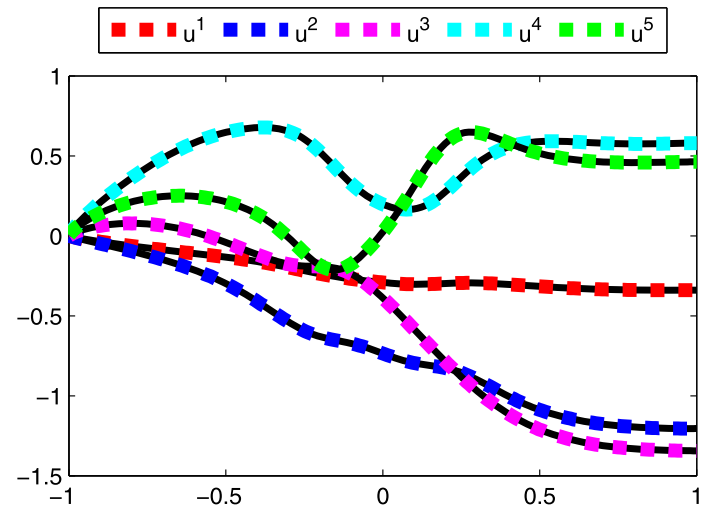

(c)

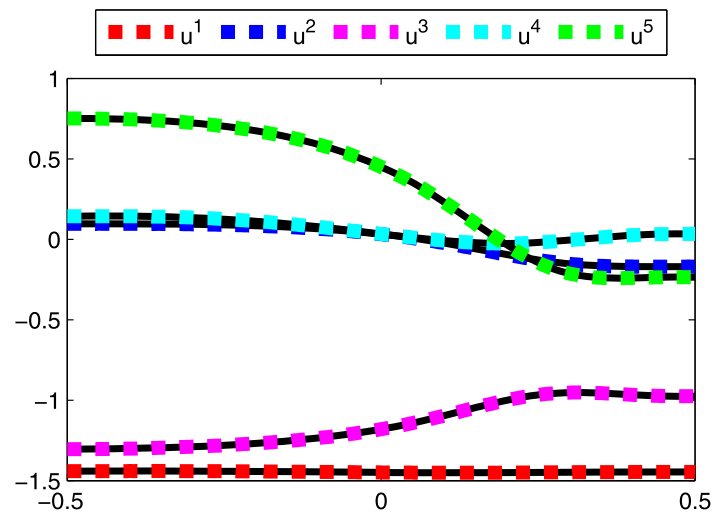

(d)

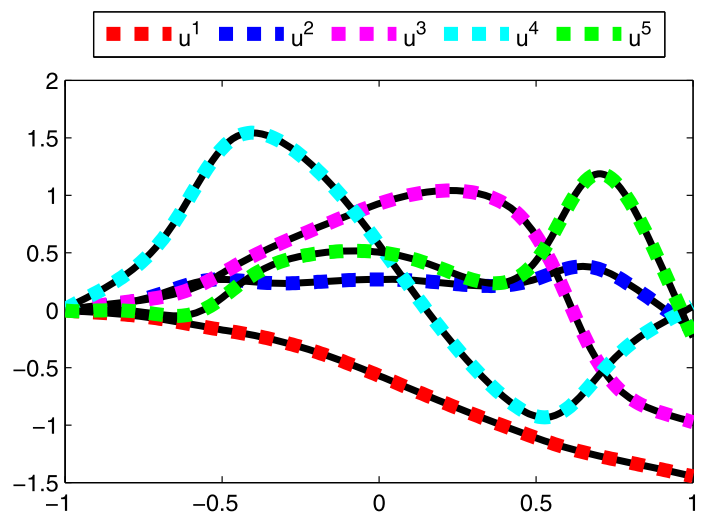

(e)

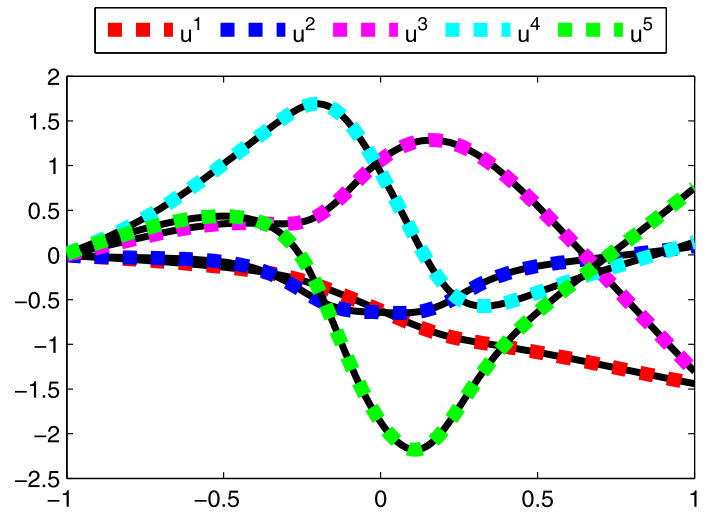

(f)

Fig. 9. (a) First components of the target $\left.\tilde{u}^{q}\right|_{\Gamma_{1}}$ (continuous black line) and of the numerical approximation $\left.u^{q}\right|_{\Gamma_{1}}$ (dashed lines); (b) first components of $\left.\tilde{u}^{q}\right|_{\Gamma_{2}}$ (continuous black line) and $\left.u^{q}\right|_{\Gamma_{2}}$ (dashed lines); (c) first components of $\left.\tilde{u}^{q}\right|_{\Gamma_{3}}$ (continuous black line) and $u^{q} \mid \Gamma_{3}$ (dashed lines); (d), (e), (f) similar results for the second components of the eigenfunctions.

boundaries of the numerical approximation $V$. In this case we obtain $J(V)=3.6 \times 10^{-3}$. In Fig. 9 we plot the traces of the two components of the eigenfunctions associated to the five eigenvalues on $\Gamma_{1}, \Gamma_{2}$ and $\Gamma_{3}$.

Note that $\Gamma_{m}$ (which is the part of the boundary where the traces of the eigenfunctions are measured) may be just a subset of $\Gamma_{N}$. Moreover, as was mentioned, typically we obtained also good results taking $N=1$. In the last example, we consider the same target configuration of the previous example, but now we take $N=1$ and the traces of the eigenfunctions are measured in $\Gamma_{m}=\Gamma_{4} \cup \Gamma_{5}$, where $\Gamma_{4}=[0.5,1] \times\{-0.5\}$ and $\Gamma_{5}=\{0.5\} \times[-0.5,0]$. In Fig. 10 we plot the boundary of the target $\tilde{V}$ with a continuous black line and the boundary of $V$ with a red dashed line. The initial guess was the same as in the previous example. The boundary $\Gamma_{m}$ is marked with a thick black line. In this example we obtain $J(V)=3.4 \times 10^{-5}$.

In Fig. 11-left we plot the traces of the two components of the eigenfunctions associated to the first eigenfunction on $\Gamma_{4}$. In the right plot of the same Figure we show similar results on $\Gamma_{5}$. 


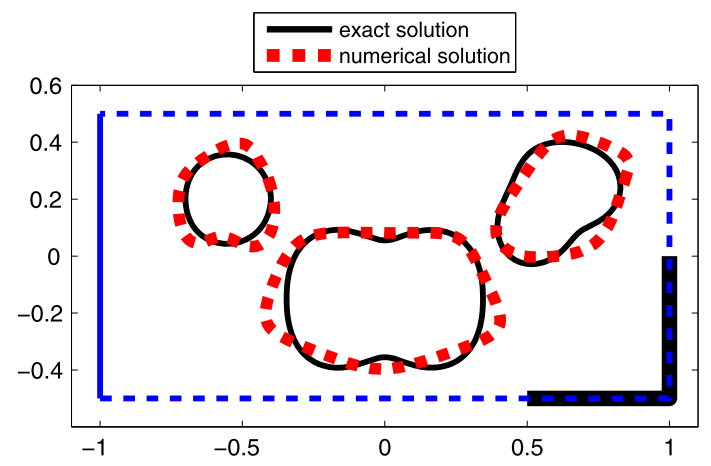

Fig. 10. The target holes $\tilde{V}$ (continuous black line) and the computed holes $V$ (red dashed line). (For interpretation of the references to color in this figure, the reader is referred to the web version of this article.)
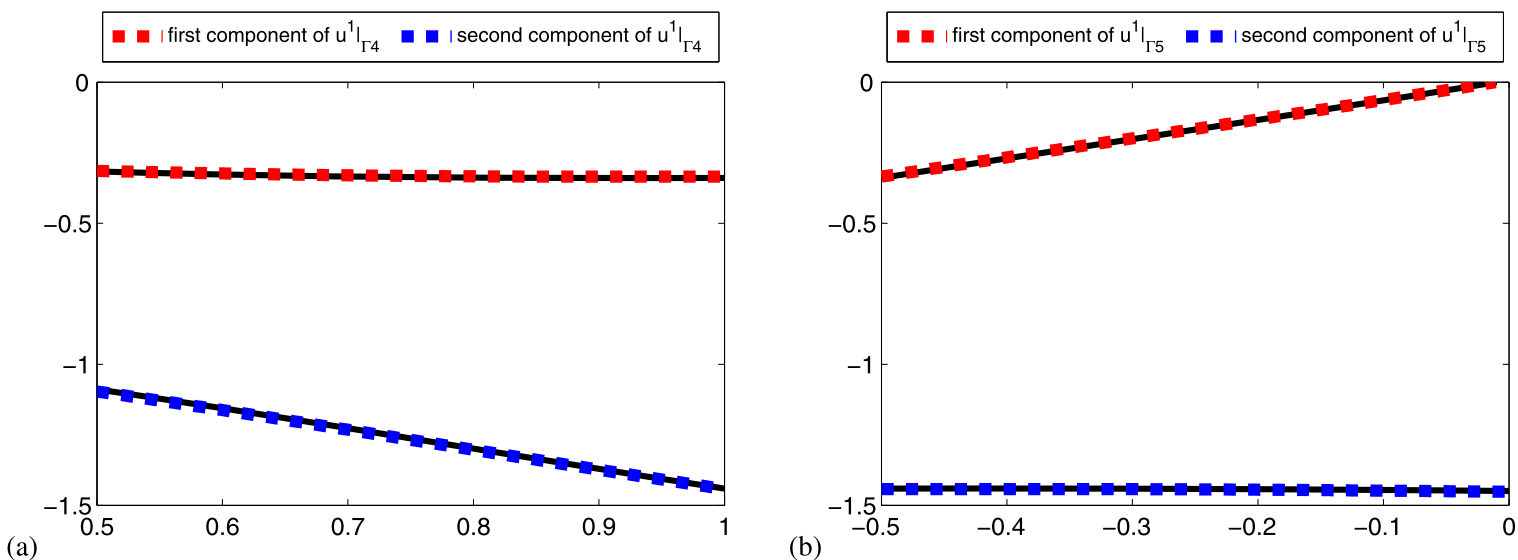

Fig. 11. (a) first and second components of the target $\left.\tilde{u}^{1}\right|_{\Gamma_{4}}$ (continuous black line), first component of $\left.u^{1}\right|_{\Gamma_{4}}$ (dashed red line) and second component of $\left.u^{1}\right|_{\Gamma_{4}}$ (dashed blue line); (b) similar results on $\Gamma_{5}$. (For interpretation of the colors in this figure, the reader is referred to the web version of this article.)

\section{Discussion}

We consider the problem of identifying the holes in an elastic domain by minimizing a functional depending on eigenvalues and traces of the corresponding eigenmodes on part of the boundary. The direct problem (the computation the eigenvalues and eigenmodes of a given domain) is solved by the Method of Fundamental Solutions. The objective functional is minimized by the BFGS method which requires the derivatives of the functional with respect to the optimization parameters, which define the shape and size of the hole(s). The shape derivative is computed by using the adjoint method; to our knowledge, this is the first time when the adjoint method is applied to a functional depending on eigenmodes (eigenvectors).

The obtained numerical results illustrate that we can obtain good reconstructions of the holes, with a few measurements of the eigenvalues and eigenmodes. However, we believe that some improvements are needed in order to have a robust nondestructive numerical method to deal with realistic problems. We use Fourier expansions to parameterize the holes. A handicap of this approach is that we cannot change the topology during the optimization procedure. In a future work we would like to address the use of a level set method coupled with the Method of Fundamental Solutions.

Another open problem is the treatment of inclusions of a different material instead of holes. In that case, instead of the Method of Fundamental Solutions, we must consider a different approach for the numerical solution of the direct problem (see e.g. [6]). Both the adjoint method and the optimization algorithm based on it would follow similar steps.

\section{References}

[1] G. Allaire, F. Jouve, A level-set method for vibration and multiple loads structural optimization, Comput. Methods Appl. Mech. Eng. 194 (2005) 3269-3290.

[2] G. Allaire, F. Jouve, A.-M. Toader, Structural optimization using sensitivity analysis and a level set method, J. Comput. Phys. 194 (1) (2004) $363-393$.

[3] C.J.S. Alves, P.R.S. Antunes, The method of fundamental solutions applied to the calculation of eigenfrequencies and eigenmodes of 2D simply connected shapes, Comput. Mater. Continua 2 (4) (2005) 251-266.

[4] C.J.S. Alves, P.R.S. Antunes, The method of fundamental solutions applied to some inverse eigenproblems, SIAM J. Sci. Comput. 35 (3) (2013) A1689-A1708. 
[5] C.J.S. Alves, N.F.M. Martins, S.S. Valtchev, Extending the method of fundamental solutions to non-homogeneous elastic wave problems, Appl. Numer. Math. (2016), http://dx.doi.org/10.1016/j.apnum.2016.06.002.

[6] P.R.S. Antunes, Is it possible to tune a drum? submitted for publication.

[7] A.H. Barnett, T. Betcke, Stability and convergence of the method of fundamental solutions for Helmholtz problems on analytic domains, J. Comput. Phys. 227 (2008) 7003-7026.

[8] T. Betcke, L.N. Trefethen, Reviving the method of particular solutions, SIAM Rev. 47 (2005) 469-491.

[9] J. Céa, Conception optimale ou identification de formes. Calcul rapide de la dérivée directionnelle de la fonction coût, RAIRO, Modélisation Math. Anal. 20 (1986) 371-402.

[10] M.C. Delfour, J.-P. Zolezio, Shapes and Geometries: Metrics, Analysis, Differential Calculus, and Optimization, Advances in Design and Control, SIAM, 2011.

[11] G. Golub, W. Kahan, Calculating the singular values and pseudo-inverse of a matrix, J. SIAM Numer. Anal. Ser. B 2 (2) (1965) 205-224.

[12] F. de Gournay, Velocity extension for the level-set method and multiple eigenvalues in shape optimization, SIAM J. Control Optim. 45 (1) (2006) 343-367.

[13] J. Hadamard, Mémoire sur le problème d'analyse relatif à l'équilibre des plaques élastiques encastrées, Bull. Soc. Math. Fr. (1907).

[14] A. Henrot, Extremum Problems for Eigenvalues of Elliptic Operators, Frontiers in Mathematics, Birkhäuser, 2006.

[15] N. Kikutchi, H.C. Cheng, Z.D. Ma, Optimal shape and topology design of vibrating structures, in: J. Herskovits (Ed.), Advances in Structural Optimization, Kluwer, 1995, pp. 189-222.

[16] V.D. Kupradze, M.A. Aleksidze, The method of fundamental equations for an approximate solution of certain boundary value problems, Comput. Math. Phys. 4 (1964) 82-126.

[17] R. Mathon, R.L. Johnson, The approximate solution of elliptic boundary-value problems by fundamental solutions, SIAM J. Numer. Anal. 14 (1977) 638-650.

[18] W. McLean, Strongly Elliptic Systems and Boundary Integral Equations, Cambridge University Press, 2000.

[19] F. Murat, J. Simon, Sur le contrôle par un domaine géométrique, Internal report n. 76 015, Laboratoire d'Analyse Numérique de l'Université Paris VI, 1976.

[20] C.M. Mota Soares, M. Moreira de Freitas, A.L. Araújo, P. Pedersen, Identification of material properties of composite plate specimens, Compos. Struct. 25 (1993) 277-285.

[21] S. Oliveira, A.M. Toader, P. Vieira, Damage identification in a concrete dam by fitting measured modal parameters, Nonlinear Anal., Real World Appl. 13 (2012) 2888-2899.

[22] S. Osher, F. Santosa, Level-set methods for optimization problems involving geometry and constraints: frequencies of a two-density inhomogeneous drum, J. Comput. Phys. 171 (2001) 272-288.

[23] O. Pironneau, Optimal Shape Design for Elliptic Systems, Springer Series in Computational Physics, 1984.

[24] B. Rousselet, D. Chenais, Continuité et différentiabilité d'éléments propres: application à l'optimisation de structures, Appl. Math. Optim. 22 (1) (1990) $27-59$.

[25] A.-M. Toader, C. Barbarosie, Optimization of eigenvalues and eigenmodes by using the adjoint method, in: Maitine Bergounioux, Édouard Oudet, Martin Rumpf, Guilaume Carlier, Thierry Champion, Filippo Santambrogio (Eds.), Topological Optimization and Optimal Transport in the Applied Sciences, in: Radon Series on Computational and Applied Mathematics, vol. 17, 2017, forthcoming.

[26] S.W. Walker, The Shapes of Things: A Practical Guide to Differential Geometry and the Shape Derivative, Advances in Design and Control, SIAM, 2015. 